\title{
Less is more in solid-dominant lung cancer? Sublobar resection versus lobectomy for solid-dominant stage IA non-small-cell lung cancer: A meta-analysis study
}

\author{
JUNTANG GUO $^{1}$, YANG LIU ${ }^{1}$, XIAODONG TIAN ${ }^{1}$, ZHIPENG REN $^{1}$, JIXING LIN ${ }^{2}$, \\ BAILIN WANG $^{2}$ and CHAOYANG LIANG ${ }^{1}$ \\ ${ }^{1}$ Department of Thoracic Surgery, Chinese PLA General Hospital, Beijing 100853; ${ }^{2}$ Department of Thoracic Surgery, \\ Hainan Branch, Chinese PLA General Hospital, Sanya, Hainan 572014, P.R. China
}

Received September 26, 2018; Accepted June 27, 2019

DOI: $10.3892 / \mathrm{mco} .2019 .1914$

\begin{abstract}
Although lobectomy is well established as the standard surgical procedure for stage IA non-small-cell lung cancer (NSCLC), sublobar resection is increasingly preferred, particularly in intentional segmentectomy for radiologically less-invasive small NSCLC. However, the indication for sublobar resection of radiologically pure solid or solid-dominant NSCLC remains controversial, owing to its invasive pathological characteristics. Therefore, the present meta-analysis was conducted to compare the efficacy of sublobar resection with lobectomy for treating solid-dominant stage IA NSCLC. An electronic search was conducted using four online databases from their dates of inception to April 2017. The hazard ratio (HR) was used as a summary statistic for censored outcomes and the odds ratio (OR) was used as the summary statistic for dichotomous variables. A total of nine studies met the selection criteria, including a total of 2,265 patients (1,728 patients underwent lobectomy, 425 segmentectomy and 112 wedge resection). From the available data, patients treated with a sublobar resection had a higher risk of local recurrence compared with patients treated with lobectomy $[\mathrm{OR}=1.89$; 95\% confidence interval (CI), 1.02-3.50; $\mathrm{P}=0.04]$. However, no obvious difference in local recurrence was found in a subgroup analysis of segmentectomy compared with lobectomy (OR=1.19; 95\% CI, 0.68-2.10; $\mathrm{P}=0.61)$. Sublobar resection was not associated with a significantly negative impact on distant recurrence $(\mathrm{OR}=1.09 ; 95 \% \mathrm{CI}, 0.55-2.16 ; \mathrm{P}=0.796)$. Patients in
\end{abstract}

Correspondence to: Professor Chaoyang Liang, Department of Thoracic Surgery, Chinese PLA General Hospital, 28 Fuxing Street, Beijing 100853, P.R. China

E-mail: guojuntang 301@sina.com

Abbreviations: NSCLC, non-small-cell lung cancer; HR, hazard ratio; OR, odds ratio; RFS, recurrence-free survival; OS, overall survival; CT, computer tomography; GGO, ground-glass opacity; DFS, disease-free survival; RCT, randomized control trial

Key words: lobectomy, non-small-cell lung cancer, sublobar resection, segmentectomy, solid the sublobar resection group had no significant differences in recurrence-free survival (RFS; HR=1.43; 95\% CI, 0.76-2.69; $\mathrm{P}=0.27)$ and overall survival $(\mathrm{OS} ; \mathrm{HR}=0.96 ; 95 \% \mathrm{CI}, 0.75-1.23$; $\mathrm{P}=0.77$ ) compared with those in the lobectomy group. In the subgroup analysis of anatomic segmentectomy compared with lobectomy, there was no significant difference in RFS, with mild inter-study heterogeneity. The current meta-analysis suggested that segmentectomy had a comparable oncologic efficacy to lobectomy for solid-dominant stage IA NSCLC. Therefore, segmentectomy may be a feasible alternative in selected cases of solid-dominant stage IA NSCLC. However, these findings should be confirmed by prospective randomized controlled trials in the future.

\section{Introduction}

Lobectomy has been the standard procedure for lung cancer resection since the widespread acceptance of the 1995 Lung Cancer Study Group (LCSG) randomized trial of lobectomy compared with limited resection for stage IA non-small-cell lung cancer (NSCLC) (1). However, lung cancer screening with low-dose computed tomography (CT) and widespread use of spiral CT imaging has contributed to the identification and diagnosis of early-stage NSCLC $(2,3)$. In the past decade, the number of patients presenting with very small and peripheral lung cancers has markedly increased. Meanwhile, a growing population of older patients with significant medical comorbidities that preclude major operations are being diagnosed with early lung cancer. These factors have led to the popularity of sublobar resection in recent years.

Early lung cancer presents as a wide area of ground-glass opacity (GGO) on CT scans, which is likely to be less invasive adenocarcinoma associated with a good prognosis (4-6). Therefore, these patients are considered to be feasible candidates for sublobar resections, i.e. segmentectomy or wedge resection, as confirmed by the prospective JCOG 0201 study in Japan (4).

However, radiologically solid-dominant lung cancer has been regarded as a different, highly invasive category of lung cancer $(7,8)$. Thus, sublobar resection for lung cancer with a solid-dominant appearance on thin-section CT scans, namely 
invasive lung cancer, remains controversial $(9,10)$. A total of three multi-center, prospective, randomized studies focused on this issue are currently ongoing, and the data have not yet been published (11-13).

Segmentectomy, rather than wedge resection, is preferred for patients with stage IA NSCLC as it is an anatomical resection involving more extensive lymph node dissection (14-16). Whether sublobar resection, particularly segmentectomy, is comparable to lobectomy in terms of oncologic outcomes in radiologically solid (i.e. invasive) NSCLC remains unknown.

This meta-analysis investigated whether sublobar resection has comparable oncologic outcomes to lobectomy in lung cancer with a solid-dominant appearance. The evaluated outcomes were local recurrence, distant recurrence, recurrence-free survival (RFS) and overall survival (OS).

\section{Materials and methods}

Search strategies. Systematic computerized searches of the PubMed, Embase and Cochrane Library databases and Google Scholar were performed from their dates of inception to April 2017. The following search terms were used: 'non-small-cell lung cancer (NSCLC)/lung cancer' and 'lobectomy/sublobectomy/segmentectomy/limited resection/sublobar resection' and 'recurrence/prognosis/survival' and 'solid'. The search was limited to English and the Abstract/Title. The citation lists of all retrieved articles were scanned to identify other potentially relevant publications.

Study selection. The following criteria were used for study inclusion. i) Either completed randomized controlled trials (RCTs) or retrospective observational studies that compared sublobar resection/segmentectomy with lobectomy in treating patients with clinical stage IA NSCLC, according to the 7th edition of TNM classification (17). ii) Nodules were peripheral with 'solid or solid-dominant appearance' on thin-section CT. The solid component was defined as an area of increased opacification that completely obscured the underlying vascular markings. GGO was defined as an area of slight, homogeneous increase in density that did not obscure the underlying vascular markings (9). In the current study, a solid tumor was defined as a tumor exhibiting only consolidation without GGO, while a solid dominant tumor was defined as a tumor in which the ratio of the maximum diameter of consolidation to the maximum tumor diameter was $>50 \%$ on thin-section CT. iii) The primary outcomes of interest in this study were OS, disease-free survival (DFS)/RFS, and local or distant recurrence rate. Only studies that reported at least one of the outcomes were included. iv) The most recent or completed study was chosen if the studies were based on overlapping patients.

The exclusion criteria were as follows: i) Stage IA lung cancer was characterized as GGO-dominant or its nature was not described on $\mathrm{CT}$; ii) papers that were not published in English; and iii) case reports, abstracts, conference reports, reviews and experiments.

Statistical analysis. The meta-analysis was performed in accordance with the recommendations of the Cochrane Collaboration and the Quality of Reporting of Meta-analyses (QUORUM) guidelines $(18,19)$. The hazard ratio (HR) was

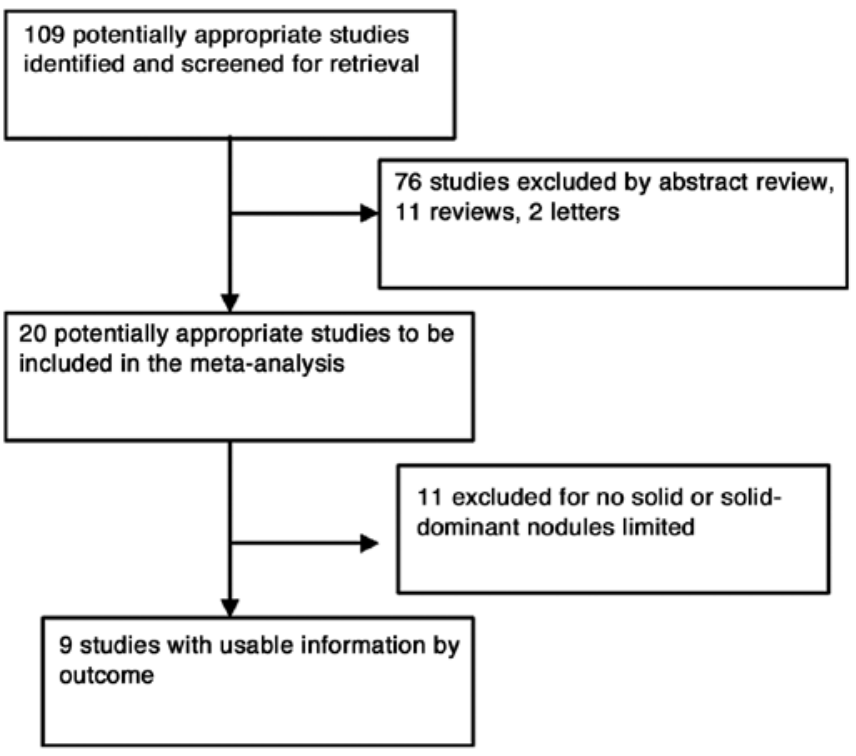

Figure 1. Flow diagram of the study selection procedure.

used as a summary statistic for censored outcomes (OS and RFS), as previously described (20). An HR $>1$ represented a survival benefit favoring the sublobar resection/segmentectomy group. The odds ratio (OR) was used as the summary statistic for dichotomous variables. An OR $<1$ favored the sublobar resection/segmentectomy group. The pooled OR/HR and $95 \%$ confidence intervals (CIs) were graphically presented as forest plots. The effect measure OR/HR was calculated by a fixed effects model (using the Mantel-Haenszel method) or a random effects model (using the DerSimonian and Laird method) based on the heterogeneity among studies $(21,22)$.

The heterogeneity of the included studies was detected using the Cochran test. Random effects models were used if high heterogeneity was detected among the studies $(\mathrm{P}<0.1$ or $I^{2}>50 \%$ ). Otherwise, fixed effects models were used. To combine the data, an HR with $95 \%$ CI was used, which were directly obtained from the original articles. When the HR was not directly reported in the original articles, it was estimated, as previously described (23). A funnel plot and the Egger test were used to investigate possible publication bias (24). Statistical significance was set at $\mathrm{P}<0.05$.

Analyses were performed using STATA version 13.0 software (Stata Corporation).

\section{Results}

Literature Search and Study Characteristics. A total of 109 publications were identified using the predefined search strategy (Fig. 1). Eighty-nine studies were excluded after screening the titles and abstracts, and full texts of the remaining 20 studies were retrieved. These included 11 reviews, two letters and 76 studies, which were either wedge resection/sublobar resection versus lobectomy or insufficient data for the specified endpoints. Eleven were excluded due to lack of definite solid or solid-dominant nodules reporting. Finally, nine studies that met the inclusion criteria were included in the meta-analysis (Fig. 1). Study characteristics are summarized in Table I. The combined study population 

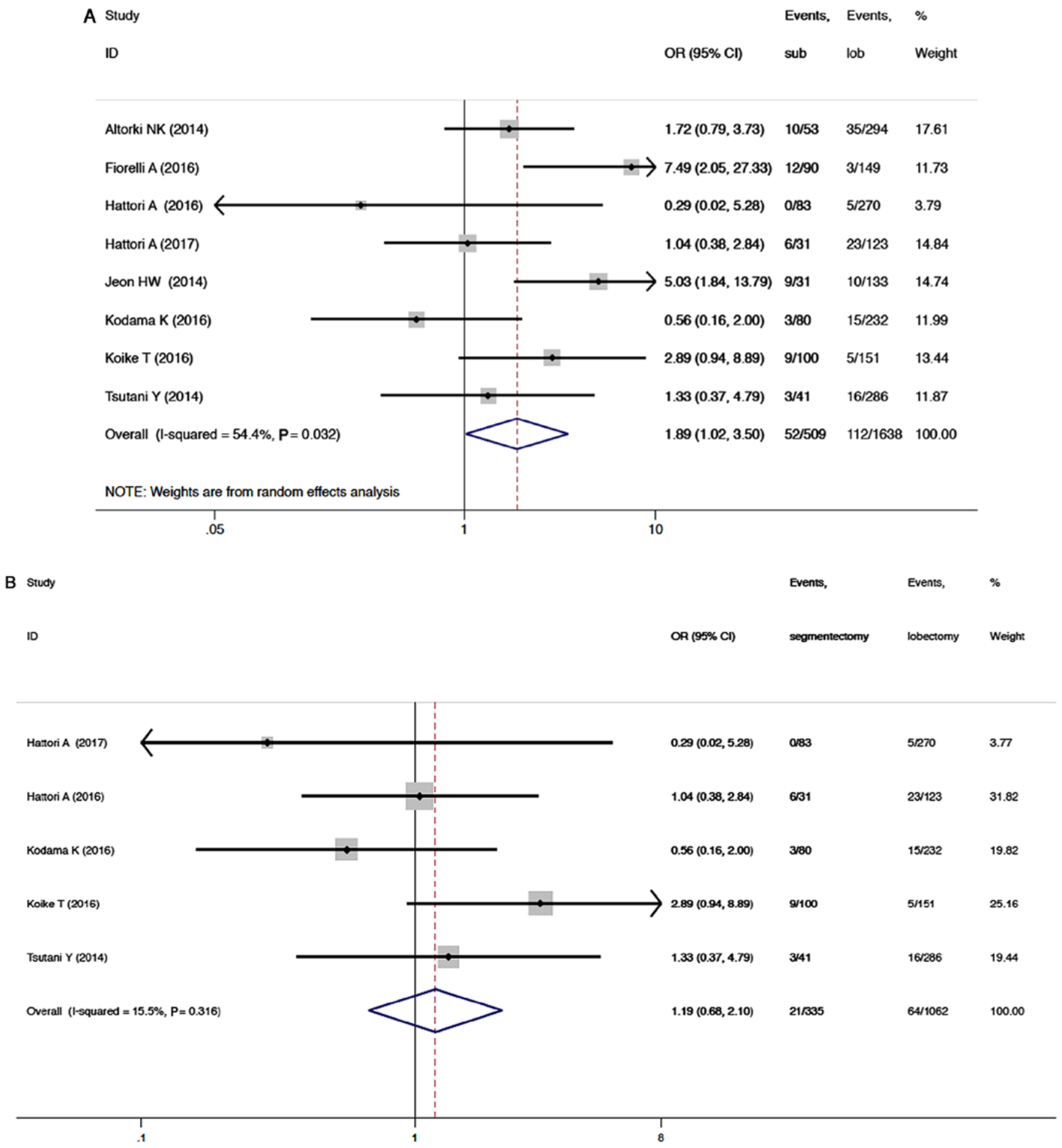

Figure 2. Local recurrence after (A) sublobar resection vs. lobectomy and (B) local recurrence after segmentectomy vs. lobectomy for early-stage solid-dominant NSCLC. Squares are point estimates of the treatment effect (HR, OR and WMD), with 95\% CIs indicated by the horizontal bars. Diamonds are the summary estimate from the pooled studies with 95\% CI. NSCLC, non-small-cell lung cancer; CI, confidence interval; HR, hazard ratio; OR, odds ratio.

from the included studies was 2,265 patients with 1,728 lobectomies, 425 segmentectomies and 112 wedge resections. All studies were retrospective studies. Seven studies reported several pathological types of NSCLC, whereas two studies only included adenocarcinoma. In four studies, sublobar resection involved segementectomy and wedge resection, but in the other five studies, sublobar resection only referred to segmentectomy. Five studies described intentional sublobar resection, three compromised procedure and one had both categories.

\section{Primary outcome measures}

Local and Distant Recurrence. Eight studies reported local recurrence in the sublobar resection and lobectomy groups, providing a total sample size of 2,147 patients for evaluation. Meta-analysis of the data showed that patients treated with a sublobar resection were inferior to patients treated with lobectomy, with a pooled OR of 1.89 (95\% CI, 1.02-3.50; $\mathrm{P}=0.04$; Fig. 2A). There was moderate inter-study heterogeneity $\left(\mathrm{P}=0.03 ; I^{2}=54 \%\right)$. 


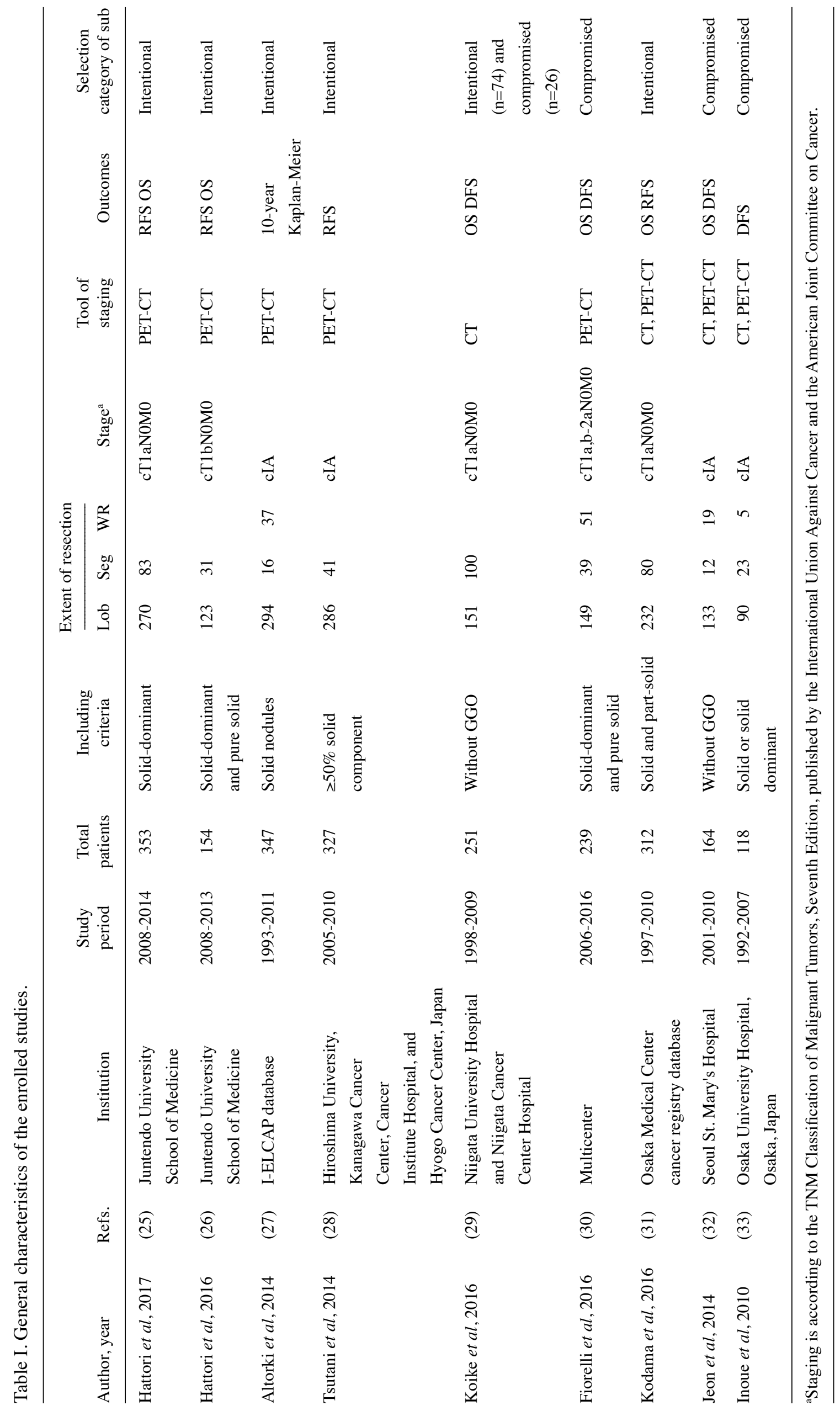




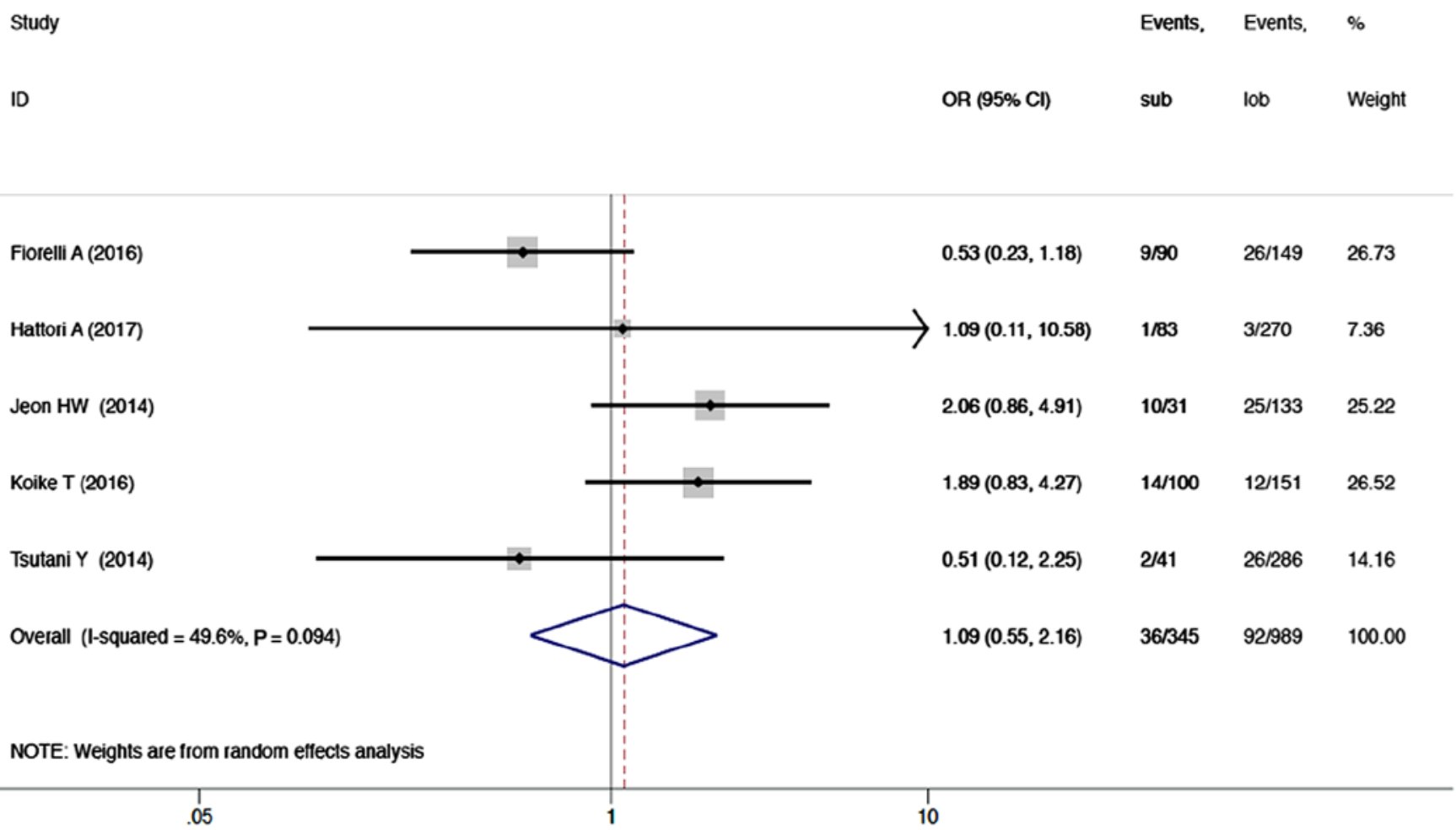

Figure 3. Distant recurrence after sublobar resection vs. lobectomy for early-stage solid-dominant NSCLC. Squares are point estimates of the treatment effect (HR, OR and WMD), with 95\% CIs indicated by the horizontal bars. Diamonds are the summary estimate from the pooled studies with $95 \%$ CI. NSCLC, non-small-cell lung cancer; CI, confidence interval; HR, hazard ratio; OR, odds ratio.

However, in subgroup analysis of anatomic segmentectomy versus lobectomy, there was no obvious difference in local recurrence between the two groups $(\mathrm{OR}=1.19$; 95\% CI, 0.68-2.10; P=0.61; Fig. 2B). Inter-study heterogeneity was not significant in subgroup analysis $\left(\mathrm{P}=0.316 ; I^{2}=15.5 \%\right)$.

Sublobar resection was not associated with a significant negative impact on distant recurrence based on five reports (1,334 patients) as compared to lobectomy. The OR was 1.09 (95\% CI, 0.55-2.16; P=0.796; $I^{2}=49.6 \%$; Fig. 3 ).

RFS and OS. For RFS, eight eligible studies that included a total of 1918 patients were pooled. Given the significant heterogeneity among studies $\left(I^{2}=74.1 \%\right)$, a random-effects model was used to pool the HR of the studies. As seen in Fig. 4A, the combined HR for the eight studies was 1.43 (95\% CI, 0.76-2.69; $\mathrm{P}=0.27$ ). Both in intentional and compromised sublobar resection groups, there are similar combined HR (1.40 vs. 1.44) in RFS. Accordingly, there was no statistical difference in RFS between sublobar resection (both intentional and compromised sublobar resection) and lobectomy group. In subgroup analysis of anatomic segmentectomy versus lobectomy, there was no statistical difference in RFS (HR=1.40; 95\% CI, 0.79-2.48; $\mathrm{P}=0.244$; Fig. 4B). Furthermore, inter-study heterogeneity was not obvious in subgroup analysis $\left(\mathrm{P}=0.253 ; I^{2}=25.2 \%\right)$.

For OS, eight studies with a total of 1938 patients were included in the quantitative analysis. The combined HR for the eight studies was 0.96 (95\% CI, 0.75-1.24; P=0.77; Fig. 5) with a random-effects model, indicating that sublobar resection was associated with similar OS as lobectomy. There was minor heterogeneity between studies $\left(I^{2}=37.3 \% ; \mathrm{P}=0.13\right)$.
Publication Bias. The results of the Egger test did not suggest any evidence of publication bias in local and distant recurrence $(\mathrm{P}=0.933, \mathrm{P}=0.699$; respectively). For RFS and OS, the funnel plots provided no evidence of overt publication bias. The Egger test also showed no significant publication bias in RFS ( $\mathrm{P}=0.774)$ and $\mathrm{OS}(\mathrm{P}=0.557)$.

\section{Discussion}

Lobectomy is widely recommended as the standard treatment for patients with stage IA NSCLC who can tolerate the procedure (1). Recently, sublobar resection (including wedge resection and segmentectomy) was suggested as an alternative surgical treatment for early-stage NSCLC (34-45). A number of meta-analyses have reported that sublobar resection or segmentectomy have comparable oncologic outcomes to lobectomy in patients with stage IA NSCLC (46-48). The most favorable subset consists of radiologically non-invasive lung cancer, usually defined as a consolidation/tumor $(\mathrm{C} / \mathrm{T})$ ratio $<0.5$ on thin-section CT $(4,49,50)$. Recently, several studies have compared the efficacy of sublobar resection with lobectomy for treating solid-dominant stage IA NSCLC, which is conventionally an unfavorable indication for sublobar resection $(51,52)$. Therefore, this meta-analysis involving nine studies and 2,265 patients was conducted to examine the efficacy of sublobar resection for the treatment of solid-dominant stage IA NSCLC.

According to this meta-analysis, sublobar resection had a higher local recurrence rate than lobectomy in solid-dominant NSCLC. However, there was no significant difference between the local recurrence rates of segmentectomy and lobectomy. 
A Study

Fiorelli A (2016)

Inoue $M(2010)$

Jeon HW (2014)

Subtotal (1-squared $=90.8 \%, P=0.000)$

2

Hattori A (2016)

Hattori A (2017)

Kodama K (2016)

Koike T (2016)

Tsutani Y (2014)

Subtotal $(1-$ squared $=25.2 \%, P=0.253)$

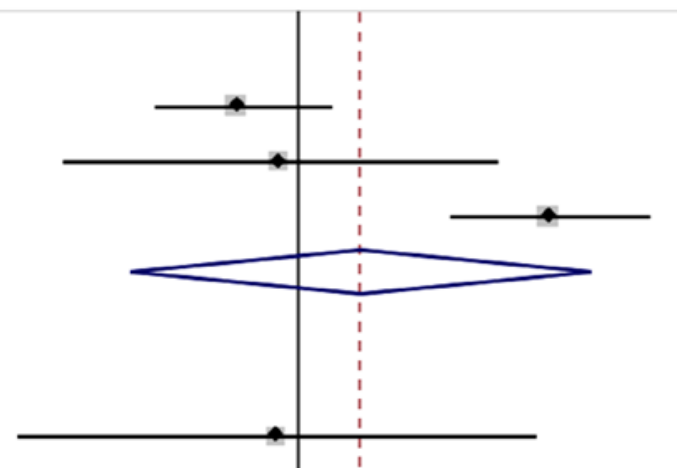

$0.70(0.43,1.22) \quad 16.23$

$0.90(0.25,3.23) \quad 10.46$

$4.39(2.44,7.89) \quad 15.77$

$1.44(0.37,5.58)$

42.46

Overall (1-squared $=74.1 \%, P=0.000$ )

NOTE: Weights are from random effects analysis

$\begin{array}{lll}1 & 1 & 1 \\ .1 & 1 & 8\end{array}$

$0.88(0.19,4.05)$

8.86

$2.92(1.12,7.69) \quad 12.80$

$1.99(0.47,6.49) \quad 10.23$

$1.50(0.56,4.23) \quad 12.47$

$0.67(0.27,1.70) \quad 13.17$

$1.40(0.79,2.48) \quad 57.54$

$1.43(0.76,2.69) \quad 100.00$

B study

ID

HR $(95 \% \mathrm{Cl})$

Weight

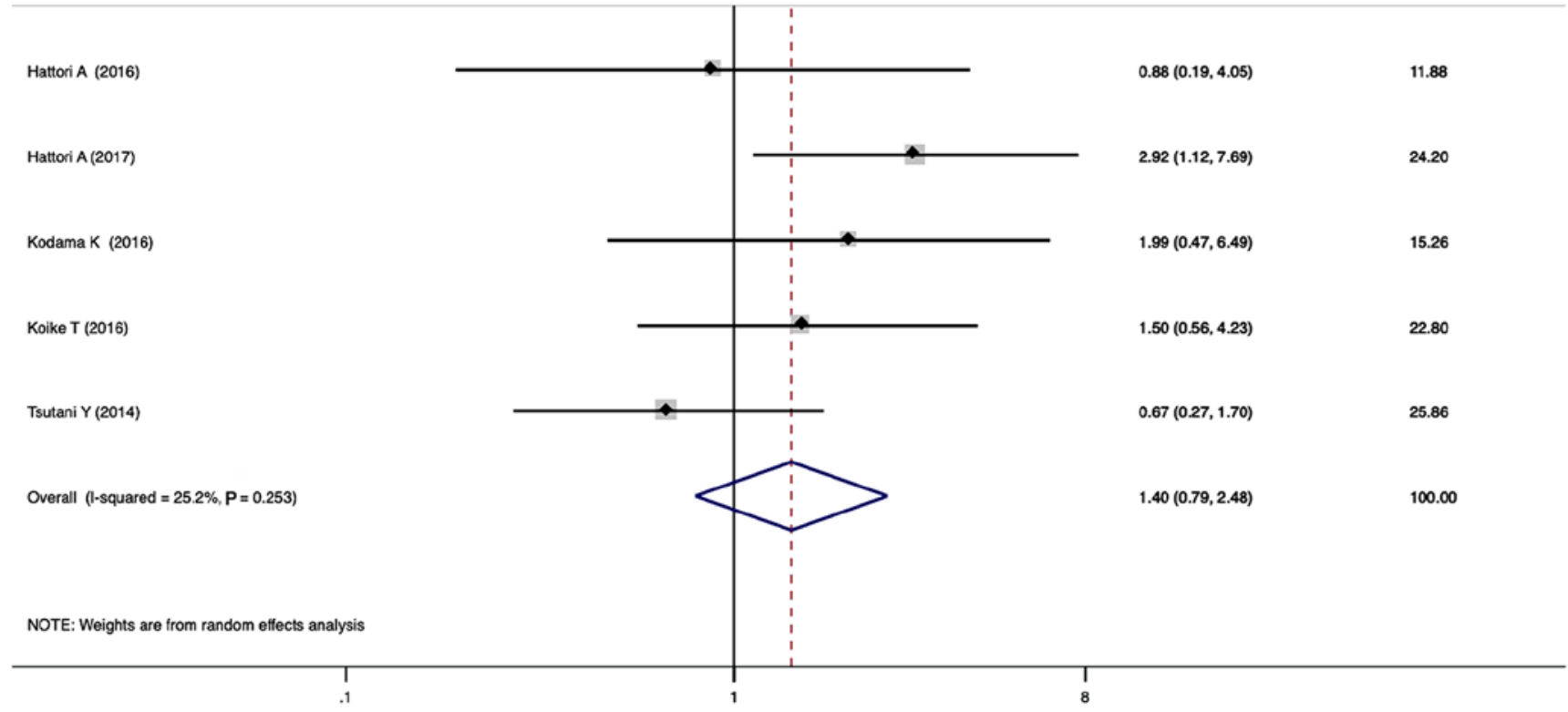

Figure 4. Recurrence-free survival after sublobar resection vs. lobectomy for early-stage solid-dominant NSCLC. (A) Compromised sublobar resection group. (B) Intentional sublobar resection group. Squares are point estimates of the treatment effect (HR, OR and WMD), with 95\% CIs indicated by the horizontal bars. Diamonds are the summary estimate from the pooled studies with 95\% CI. NSCLC, non-small-cell lung cancer; CI, confidence interval; HR, hazard ratio; OR, odds ratio.

Moreover, the distant recurrence risk was comparable between sublobar resection and lobectomy. The RFS and OS values of patients with solid-dominant tumors who underwent sublobar resection were similar to those of patients who underwent 


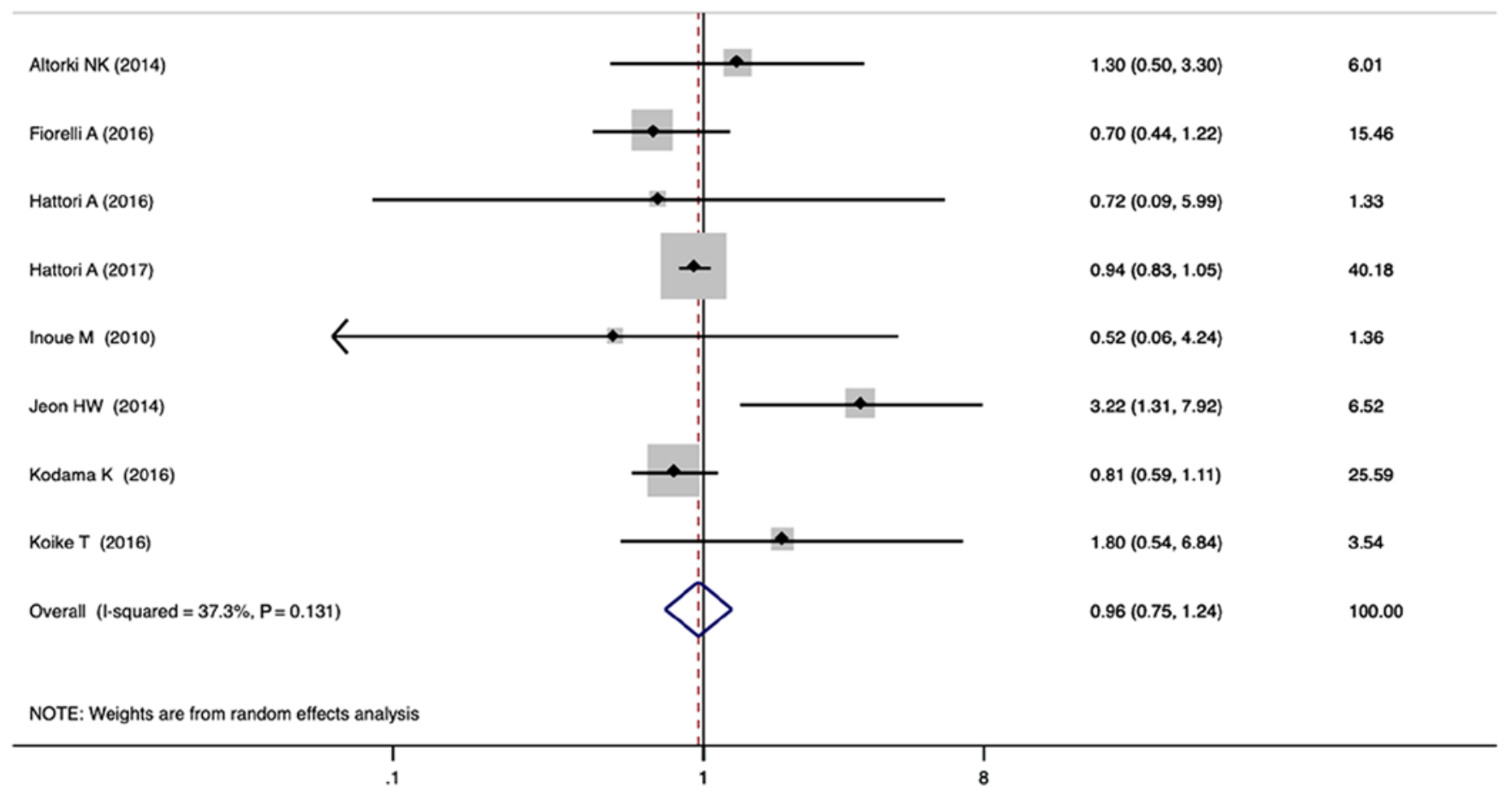

Figure 5. Overall survival after sublobar resection vs. lobectomy for early-stage solid-dominant NSCLC. Squares are point estimates of the treatment effect (HR, OR and WMD), with 95\% CIs indicated by the horizontal bars. Diamonds are the summary estimate from the pooled studies with 95\% CI. NSCLC, non-small-cell lung cancer; $\mathrm{CI}$, confidence interval; HR, hazard ratio; OR, odds ratio.

lobectomy. Despite the obvious heterogeneity between studies in RFS, studies with intentional sublobar resection had no obvious heterogeneity. The subgroup analysis showed that segmentectomy was equivalent to lobectomy with respect to RFS, with no obvious heterogeneity. The results indicated that anatomic segmentectomy with systemic lymph node dissection may be an alternative surgical procedure for solid-dominant stage IA NSCLC. These findings should be confirmed in prospective studies, such as JCOG 0802/WJOG4607L $(11,12)$ and NCT00499330 (13).

In comparing with other meta-analyses, the present study selected patients with radiologically solid-dominant early stage NSCLC. Up to now, the use of segmentectomy in radiologically solid-dominant early stage NSCLC has been controversial. Given the lack of solid evidence, it is necessary to summarize the relevant studies prior to the results of several large RCTs being delivered. This analysis suggested segmentectomy had comparable oncologic efficacy to lobectomy for solid-dominant stage IA NSCLC. This may be a novel concept.

The present meta-analysis had several limitations. First, the meta-analysis was based on retrospective cohort studies and the level of evidence was relatively low compared with that for RCTs. The number of studies was limited. In addition, not all studies were of high quality, which introduced a potential bias. Second, even though subgroup analyses were conducted, heterogeneity persisted in the meta-analysis, primarily due to the use of wedge resection in four studies. Numerous studies have highlighted the technical and oncological differences between wedge resection and segmentectomy (14-16). Segmentectomy is an anatomic resection frequently accompanied by hilar and mediastinal lymph node sampling or dissection. It is inappropriate to combine the two oncologically-distinct procedures of wedge resection and segmentectomy in radiologically-invasive stage IA lung cancer. Moreover, heterogeneity was also observed in solid part ratio (pure solid or solid-dominant type), tumor size (T1a or T1b) and the accuracy of clinical staging. Third, publication bias is a major concern in all meta-analyses. Although the present analysis did not show publication bias, it should be noted that this meta-analysis could not completely exclude biases. For example, intentional sublobar resection had a different compromised selection criteria, which may lead to bias. Finally, some of the included studies reported a relatively short follow-up duration. Therefore, RCTs with longer follow-up durations are needed to precisely compare segmentectomy (not including wedge resection) with lobectomy in solid-dominant stage IA NSCLC.

The current meta-analysis suggested that lobectomy and sublobar resection for solid-dominant stage IA NSCLC were equivalent with respect to distant recurrence, RFS and OS, but the outcome for local recurrence with sublobar resection was inferior to that with lobectomy. Nevertheless, segmentectomy had comparable oncologic efficacy to lobectomy for solid-dominant stage IA NSCLC. Therefore, segmentectomy with systemic node dissection/sampling may be a feasible alternative in selected solid-dominant (not pure-solid) stage IA NSCLC cases, such as smaller 
peripheral neoplastic nodules (53), air bronchogram (54) and lower SUVmax $(10,55,56)$. However, these findings should be confirmed by prospective randomized controlled trials in the future.

\section{Acknowledgements}

Not applicable.

\section{Funding}

No funding was received.

\section{Availability of data and materials}

The datasets used and/or analyzed during the current study are available from the corresponding author on reasonable request.

\section{Authors' contributions}

JG participated in the literature search, performed the statistical analysis, and drafted the manuscript. ZR, JL and BW participated in the literature search. YL and CL participated in the study design. XT conceived the study, participated in its design and coordination, and helped to draft the manuscript. All authors read and approved the final manuscript.

\section{Ethics approval and consent to participate}

Not applicable.

\section{Patient consent for publication}

Not applicable.

\section{Competing interests}

The authors declare that they have no competing interests.

\section{References}

1. Ginsberg RJ and Rubinstein LV; Lung Cancer Study Group Randomized trial of lobectomy versus limited resection for T1 N0 non-small cell lung cancer. Ann Thorac Surg 60: 615-622, discussion 622-623, 1995 .

2. Church TR, Black WC, Aberle DR, Berg CD, Clingan KL, Duan F, Fagerstrom RM, Gareen IF, Gierada DS, Jones GC, et al National Lung Screening Trial Research Team: Results of initial low-dose computed tomographic screening for lung cancer. N Engl J Med 368: 1980-1991, 2013.

3. Horeweg N, Scholten ET, de Jong PA, van der Aalst CM, Weenink C, Lammers JW, Nackaerts K, Vliegenthart R, ten Haaf K, Yousaf-Khan UA, et al: Detection of lung cancer through low-dose CT screening (NELSON): A prespecified analysis of screening test performance and interval cancers. Lancet Oncol 15: 1342-1350, 2014

4. Asamura H, Hishida T, Suzuki K, Koike T, Nakamura K, Kusumoto M, Nagai $\mathrm{K}$, Tada $\mathrm{H}$, Mitsudomi T, Tsuboi M, et al; Japan Clinical Oncology Group Lung Cancer Surgical Study Group: Radiographically determined noninvasive adenocarcinoma of the lung: Survival outcomes of Japan Clinical Oncology Group 0201. J Thorac Cardiovasc Surg 146: 24-30, 2013.

5. Cho JH, Choi YS, Kim J, Kim HK,Zo JI and Shim YM: Long-term outcomes of wedge resection for pulmonary ground-glass opacity nodules. Ann Thorac Surg 99: 218-222, 2015.
6. Tsutani Y, Miyata Y, Nakayama H, Okumura S, Adachi S, Yoshimura $\mathrm{M}$ and Okada M: Appropriate sublobar resection choice for ground glass opacity-dominant clinical stage IA lung adenocarcinoma: Wedge resection or segmentectomy. Chest 145: 66-71, 2014

7. Tsutani Y, Miyata Y, Yamanaka T, Nakayama H, Okumura S, Adachi S, Yoshimura M and Okada M: Solid tumors versus mixed tumors with a ground-glass opacity component in patients with clinical stage IA lung adenocarcinoma: Prognostic comparison using high-resolution computed tomography findings. J Thorac Cardiovasc Surg 146: 17-23, 2013.

8. Matsunaga T, Suzuki K, Takamochi K and Oh S: What is the radiological definition of part-solid tumour in lung cancer?. Eur J Cardiothorac Surg 51: 242-247, 2017.

9. Hattori A, Matsunaga T, Hayashi T, Takamochi K, Oh S and Suzuki K: Prognostic impact of the findings on thin-section computed tomography in patients with subcentimeter non-small cell lung cancer. J Thorac Oncol 12: 954-962, 2017.

10. Hattori A, Suzuki K, Matsunaga T, Miyasaka Y, Takamochi K and $\mathrm{Oh} \mathrm{S}$ : What is the appropriate operative strategy for radiologically solid tumours in subcentimetre lung cancer patients? Eur J Cardiothorac Surg 47: 244-249, 2015.

11. Nakamura K, Saji H, Nakajima R, Okada M, Asamura H, Shibata T, Nakamura S, Tada $\mathrm{H}$ and Tsuboi M: A phase III randomized trial of lobectomy versus limited resection for small-sized peripheral non-small cell lung cancer (JCOG0802/ WJOG4607L). Jpn J Clin Oncol 40: 271-274, 2010.

12. Yang F, Sui X, Chen X, Zhang L, Wang X, Wang S and Wang J: Sublobar resection versus lobectomy in Surgical Treatment of Elderly Patients with early-stage non-small cell lung cancer (STEPS): Study protocol for a randomized controlled trial. Trials 17: 191, 2016.

13. ClinicalTrials.gov: Comparison of different types of surgery in treating patients with stage IA non-small cell lung cancer. https://clinicaltrials.gov/ct2/show/NCT00499330?term, 2018.

14. Tamura M, Matsumoto I, Takata M, Yoshida S and Saito D: Sublobar resections in stage IA non-small-cell lung cancer: Segmentectomy versus wedge resection. Indian $\mathbf{J}$ Thorac Cardiovasc Surg 30: 264-271, 2014.

15. Sienel W, Dango S, Kirschbaum A, Cucuruz B, Hörth W, Stremmel C and Passlick B: Sublobar resections in stage IA non-small cell lung cancer: Segmentectomies result in significantly better cancer-related survival than wedge resections. Eur J Cardiothorac Surg 33: 728-734, 2008.

16. Smith CB, Swanson SJ, Mhango G and Wisnivesky JP: Survival after segmentectomy and wedge resection in stage I non-small-cell lung cancer. J Thorac Oncol 8: 73-78, 2013.

17. Goldstraw P, Crowley J, Chansky K, Giroux DJ, Groome PA, Rami-Porta R, Postmus PE, Rusch V, Sobin L; International Association for the Study of Lung Cancer International Staging Committee; Participating Institutions. The IASLC Lung Cancer Staging Project: proposals for the revision of the TNM stage groupings in the forthcoming (seventh) edition of the TNM Classification of malignant tumours. J Thorac Oncol 2: 706-714, 2007.

18. Jadad AR, Cook DJ, Jones A, Klassen TP, Tugwell P, Moher M and Moher D: Methodology and reports of systematic reviews and meta-analyses: A comparison of Cochrane reviews with articles published in paper-based journals. JAMA 280: 278-280, 1998.

19. Stroup DF, Berlin JA, Morton SC, Olkin I, Williamson GD, Rennie D, Moher D, Becker BJ, Sipe TA and Thacker SB: Meta-analysis of observational studies in epidemiology: A proposal for reporting. Meta-analysis Of Observational Studies in Epidemiology (MOOSE) group. JAMA 283: 2008-2012, 2000.

20. Siannis F, Barrett JK, Farewell VT and Tierney JF: One-stage parametric meta-analysis of time-to-event outcomes. Stat Med 29: 3030-3045, 2010.

21. DerSimonian R and Laird N: Meta-analysis in clinical trials. Control Clin Trials 7: 177-188, 1986.

22. Mantel $\mathrm{N}$ and Haenszel W: Statistical aspects of the analysis of data from retrospective studies of disease. J Natl Cancer Inst 22: 719-748, 1959.

23. Parmar MK, Torri V and Stewart L: Extracting summary statistics to perform meta-analyses of the published literature for survival endpoints. Stat Med 17: 2815-2834, 1998.

24. Egger M, Davey Smith G, Schneider M and Minder C: Bias in meta-analysis detected by a simple, graphical test. BMJ 315: 629-634, 1997.

25. Hattori A, Matsunaga T, Takamochi K, Oh S and Suzuki K: Locoregional recurrence after segmentectomy for clinical-T1aN0M0 radiologically solid non-small-cell lung carcinoma. Eur J Cardiothorac Surg 51: 518-525, 2017. 
26. Hattori A, Matsunaga T, Takamochi K, Oh S and Suzuki K: The oncological outcomes of segmentectomy in clinical-T1b lung adenocarcinoma with a solid-dominant appearance on thin-section computed tomography. Surg Today 46: 914-921, 2016.

27. Altorki NK, Yip R, Hanaoka T, Bauer T, Aye R, Kohman L, Sheppard B, Thurer R, Andaz S, Smith M, et al; I-ELCAP Investigators: Sublobar resection is equivalent to lobectomy for clinical stage 1A lung cancer in solid nodules. J Thorac Cardiovasc Surg 147: 754-764, 2014

28. Tsutani Y, Miyata Y, Nakayama H, Okumura S, Adachi S, Yoshimura $M$ and Okada M: Segmentectomy for clinical stage IA lung adenocarcinoma showing solid dominance on radiology. Eur J Cardiothorac Surg 46: 637-642, 2014.

29. Koike T, Kitahara A, Sato S, Hashimoto T, Aoki T, Koike T, Yoshiya K, Toyabe $\mathrm{S}$ and Tsuchida M: Lobectomy versus segmentectomy in radiologically pure solid small-sized non-small cell lung cancer. Ann Thorac Surg 101: 1354-1360, 2016.

30. Fiorelli A, Caronia FP, Daddi N, Loizzi D, Ampollini L, Ardò N, Ventura L, Carbognani P, Potenza R, Ardissone F, et al: Sublobar resection versus lobectomy for stage I non-small cell lung cancer: An appropriate choice in elderly patients? Surg Today 46: $1370-1382,2016$.

31. Kodama K, Higashiyama M, Okami J, Tokunaga T, Imamura F, Nakayama T, Inoue A and Kuriyama K: Oncologic outcomes of segmentectomy versus lobectomy for clinical T1a N0 M0 non-small cell lung cancer. Ann Thorac Surg 101: 504-511, 2016.

32. Jeon HW, Kim YD, Kim KS, Sung SW, Park HJ and Park JK: Sublobar resection versus lobectomy in solid-type, clinical stage IA, non-small cell lung cancer. World J Surg Oncol 12: 215, 2014.

33. Inoue M, Minami M, Sawabata N, Utsumi T, Kadota Y, Shigemura N and Okumura M: Clinical outcome of resected solid-type small-sized c-stage IA non-small cell lung cancer. Eur J Cardiothorac Surg 37: 1445-1449, 2010.

34. El-Sherif A, Gooding WE, Santos R, Pettiford B, Ferson PF Fernando HC, Urda SJ, Luketich JD and Landreneau RJ: Outcomes of sublobar resection versus lobectomy for stage I non-small cell lung cancer: A 13-year analysis. Ann Thorac Surg 82: 408-416, 2006

35. Hamatake D, Yoshida Y, Miyahara S, Yamashita S, Shiraishi T and Iwasaki A: Surgical outcomes of lung cancer measuring less than $1 \mathrm{~cm}$ in diameter. Interact Cardiovasc Thorac Surg 15 : 854-858, 2012

36. Jiang W, Pang X, Xi J, Chen X, Wang Q, Qian C and Fan H: Clinical outcome of subcentimeter non-small cell lung cancer after surgical resection: Single institution experience of 105 patients. J Surg Oncol 110: 233-238, 2014

37. Kates M, Swanson S and Wisnivesky JP: Survival following lobectomy and limited resection for the treatment of stage I non-small cell lung cancer $<=1 \mathrm{~cm}$ in size: A review of SEER data. Chest 139: 491-496, 2011.

38. Kilic A, Schuchert MJ, Pettiford BL, Pennathur A, Landreneau JR, Landreneau JP, Luketich JD and Landreneau RJ: Anatomic segmentectomy for stage I non-small cell lung cancer in the elderly. Ann Thorac Surg 87: 1662-1668, 2009.

39. Koike T, Koike T, Sato S, Hashimoto T, Aoki T, Yoshiya K, Yamato Y, Watanabe T, Akazawa K, Toyabe SI, et al; Niigata Chest Surgery Research Group: Lobectomy and limited resection in small-sized peripheral non-small cell lung cancer. J Thorac Dis 8: 3265-3274, 2016.

40. Landreneau RJ, Normolle DP, Christie NA, Awais O, Wizorek JJ, Abbas G, Pennathur A, Shende M, Weksler B, Luketich JD, et al Recurrence and survival outcomes after anatomic segmentectomy versus lobectomy for clinical stage I non-small-cell lung cancer: A propensity-matched analysis. J Clin Oncol 32: 2449-2455, 2014.

41. Okada M, Koike T, Higashiyama M, Yamato Y, Kodama K and Tsubota N: Radical sublobar resection for small-sized non-small cell lung cancer: A multicenter study. J Thorac Cardiovasc Surg 132: 769-775, 2006.

42. Okami J, Ito Y, Higashiyama M, Nakayama T, Tokunaga T, Maeda $J$ and Kodama K: Sublobar resection provides an equivalent survival after lobectomy in elderly patients with early lung cancer. Ann Thorac Surg 90: 1651-1656, 2010.
43. Tsutani Y, Miyata Y, Nakayama H, Okumura S, Adachi S, Yoshimura $\mathrm{M}$ and Okada M: Oncologic outcomes of segmentectomy compared with lobectomy for clinical stage IA lung adenocarcinoma: Propensity score-matched analysis in a multicenter study. J Thorac Cardiovasc Surg 146: 358-364, 2013.

44. Yano M, Yoshida J, Koike T, Kameyama K, Shimamoto A, Nishio W, Yoshimoto K, Utsumi T, Shiina T, Watanabe A, et al; Japanese Association for Chest Surgery: Survival of 1737 lobectomy-tolerable patients who underwent limited resection for cStage IA non-small-cell lung cancer. Eur J Cardiothorac Surg 47: 135-142, 2015 .

45. Zhao ZR, Situ DR, Lau RW, Mok TSK, Chen GG, Underwood MJ and Ng CS: Comparison of Segmentectomy and Lobectomy in Stage IA Adenocarcinomas. J Thorac Oncol 12: 890-896, 2017.

46. Zhang L, Li M, Yin R, Zhang Q and Xu L: Comparison of the oncologic outcomes of anatomic segmentectomy and lobectomy for early-stage non-small cell lung cancer. Ann Thorac Surg 99: 728-737, 2015.

47. Fan J, Wang L, Jiang GN and Gao W: Sublobectomy versus lobectomy for stage I non-small-cell lung cancer, a meta-analysis of published studies. Ann Surg Oncol 19: 661-668, 2012.

48. Cao C, Chandrakumar D, Gupta S, Yan TD and Tian DH: Could less be more?-A systematic review and meta-analysis of sublobar resections versus lobectomy for non-small cell lung cancer according to patient selection. Lung Cancer 89: 121-132, 2015.

49. Suzuki K, Koike T, Asakawa T, Kusumoto M, Asamura H, Nagai K, Tada H, Mitsudomi T, Tsuboi M, Shibata T, et al; Japan Lung Cancer Surgical Study Group (JCOG LCSSG): A prospective radiological study of thin-section computed tomography to predict pathological noninvasiveness in peripheral clinical IA lung cancer (Japan Clinical Oncology Group 0201). J Thorac Oncol 6: 751-756, 2011.

50. Razi SS, John MM, Sainathan S and Stavropoulos C: Sublobar resection is equivalent to lobectomy for T1a non-small cell lung cancer in the elderly: A Surveillance, Epidemiology, and End Results database analysis. J Surg Res 200: 683-689, 2016.

51. Sawabata N: Is segmentectomy suitable for solid-type lung cancer? J Thorac Cardiovasc Surg 146: 728-729, 2013.

52. Baisi A, Raveglia F, De Simone M and Cioffi U: Do tumor size and carcinoembryonic antigen level affect surgical management of partially solid early-stage lung cancer? Ann Thorac Surg 103: 1036, 2017

53. Hattori A, Maeyashiki T, Matsunaga T, Takamochi K, Oh S and Suzuki K: Predictors of pathological non-invasive lung cancer with pure-solid appearance on computed tomography to identify possible candidates for sublobar resection. Surg Today 46: 102-109, 2016.

54. Hattori A, Suzuki K, Maeyashiki T, Fukui M, Kitamura Y, Matsunaga T, Miyasaka Y, Takamochi K and Oh S: The presence of air bronchogram is a novel predictor of negative nodal involvement in radiologically pure-solid lung cancer. Eur J Cardiothorac Surg 45: 699-702, 2014.

55. Okada M, Mimae T, Tsutani Y, Nakayama H, Okumura S, Yoshimura M and Miyata Y: Segmentectomy versus lobectomy for clinical stage IA lung adenocarcinoma. Ann Cardiothorac Surg 3: 153-159, 2014.

56. Okada M, Nakayama H, Okumura S, Daisaki H, Adachi S, Yoshimura M and Miyata Y: Multicenter analysis of high-resolution computed tomography and positron emission tomography/computed tomography findings to choose therapeutic strategies for clinical stage IA lung adenocarcinoma. J Thorac Cardiovasc Surg 141: 1384-1391, 2011.

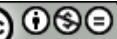

This work is licensed under a Creative Commons Attribution-NonCommercial-NoDerivatives 4.0 International (CC BY-NC-ND 4.0) License. 